\title{
CONTROLADOR ROBUSTO LMI PARA UN HELICÓPTERO DE DOS GRADOS DE LIBERTAD
}

\author{
(Robust Controller LMI for helicopter with two degrees of freedom)
}

Oscar Iván Higuera-Martínez*, Juan Mauricio Salamanca**

*Escuela de Ingeniería Electrónica, UPTC. Grupo DSP-UPTC. oscar.higuera@uptc.edu.co **Escuela de Ingeniería Electrónica, UPTC. Grupo DSP-UPTC. juan.salamanca@uptc.edu.co.

(Recibido marzo 28 de 2015 y aceptado mayo 6 de 2015)

\section{Resumen:}

Este artículo presenta el diseño de un controlador robusto Ho usando técnicas de desigualdades matriciales lineales (LMI), para controlar la posición de Pitch y de Yaw en un helicóptero. Se presenta el diseño de un controlador FF+LMI con el propósito de conseguir la estabilización del sistema, y adicionalmente se realiza el diseño de un controlador $\mathrm{FF}+\mathrm{LMI}+$ Integrador, para hacer que el error de seguimiento sea igual a cero. Posteriormente se presentan los resultados de las simulaciones sobre el modelo no lineal del sistema, así como una comparación con los controladores $\mathrm{FF}+\mathrm{LQR}$ y $\mathrm{FF}+\mathrm{LQR}+\mathrm{I}$ desarrollados por los fabricantes del dispositivo.

Palabras clave: control robusto, desigualdades matr. iciales lineales, modelo nominal, sistema no lineal.

\section{INTRODUCCIÓN}

El helicóptero experimental Quanser ${ }^{\circledR}$ de dos grados de libertad consiste en el modelo de un helicóptero montado en una base fija con dos hélices que se manejan por motores DC (ver Figura 1). La hélice derecha o delantera controla la elevación de la nariz del helicóptero sobre el eje horizontal, y la hélice izquierda o trasera controla la dirección.

El sistema Quanser documentado en este artículo es intrínsecamente inestable y por tanto se necesita aplicar la teoría de control realimentado para estabilizar el sistema. A fin de realizar esto se propone diseñar una estrategia de control robusto para estabilizar el sistema utilizando técnicas LMI (Linear Matrix Inequality).

\section{Abstract:}

This article presents the design of a robust $\mathrm{H} \infty$ controller using techniques of linear matrix inequalities (LMIs) for controlling the position of Pitch and Yaw in a helicopter. Designing a FF + LMI controller is presented for stabilizing the system, and further the design of LMI $+\mathrm{FF}+$ integrator controller is performed to make the tracking error equal to zero. Thereafter the results of simulations are showed on the nonlinear system model and a comparison with $\mathrm{FF}+\mathrm{LQR}$ and $\mathrm{FF}+\mathrm{LQR}+\mathrm{I}$ which were developed by device manufacturers.

Keywords: robust control, linear matrix inequalities, nominal model, nonlinear systems.

Adicionalmente, la posición Pitch es regulada utilizando un lazo de realimentación feed-forward que compensa el torque gravitacional $\left(\boldsymbol{\tau}_{\mathrm{g}}\right)$ (Salamanca, Díaz \& Higuera, 2007).

El diseño de controladores LMI está basado en los modelos lineales del sistema y en el problema de optimización, para encontrar una solución al problema planteado, logrando así la estabilidad y robustez del sistema.

Existen ejemplos de la aplicabilidad de diferentes tipos de control sobre este sistema, en algunos ejemplos (Guzmán, Cardozo \& Cárdenas, 2014) se presenta el diseño empleando técnicas PID y lógica difusa. En otros (Ibáñez, 2014) se indica el modelado, la identificación, la 
simulación y el control óptimo de un helicóptero con dos grados de libertad. En Bedoya y Marín (2014) se muestra la construcción y control adaptativo de un helicóptero de dos grados de libertad, y en Carrillo et al. (2015) se da la sintonización de controladores Pareto-óptimo robustos para sistemas multivariables, aplicado a este sistema.

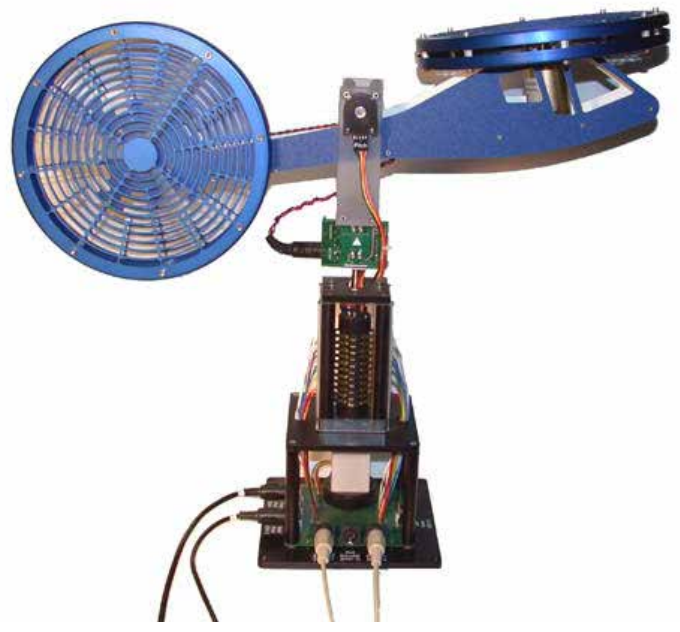

Figura. 1. Helicóptero experimental Quanser de dos grados de libertad.

Fuente: página web de quanser.com

\section{MODELADO DEL SISTEMA}

El helicóptero de dos grados de libertad (Figura 1) montado sobre el pívot por el eje del ángulo de Pitch $(\theta)$ y por el eje del ángulo de Yaw $(\psi)$, como es mostrado en la Figura 2. El Pitch es definido positivo cuando la nariz del helicóptero sube, y el Yaw es definido positivo para un giro en el sentido de las manecillas del reloj. Hay una fuerza de empuje Fp que actúa en el eje del Pitch, que es normal a la hélice delantera, y una fuerza de empuje Fy que actúa en el eje del Yaw, que es normal a la hélice trasera (Salamanca et al., 2007).

Por consiguiente, un torque de Pitch está aplicado a una distancia rp del eje de Pitch y un torque de Yaw está aplicado a una distancia ry del eje de Yaw. La fuerza gravitatoria $(\mathrm{Fg})$ genera una torque en el centro de masa del helicóptero que hala hacia abajo la nariz del helicóptero. Como es mostrado en la Figura 2, el centro de masa está a $1 \mathrm{~cm}$ de distancia del eje de pívot a lo largo de la longitud del helicóptero.

El modelo trabajado en Salamanca et al., (2007) está descrito por las ecuaciones de movimientos, las cuales se encuentran usando el método del Lagrangiano. Posteriormente se hallan las cinemáticas del centro de masa del helicóptero. Las cinemáticas se usan para encontrar las energías potencial y cinética, involucradas en el sistema del helicóptero. Posteriormente se usa el método de Euler-Lagrange para derivar las ecuaciones no lineales que describen los movimientos del helicóptero, obteniendo finalmente (1).

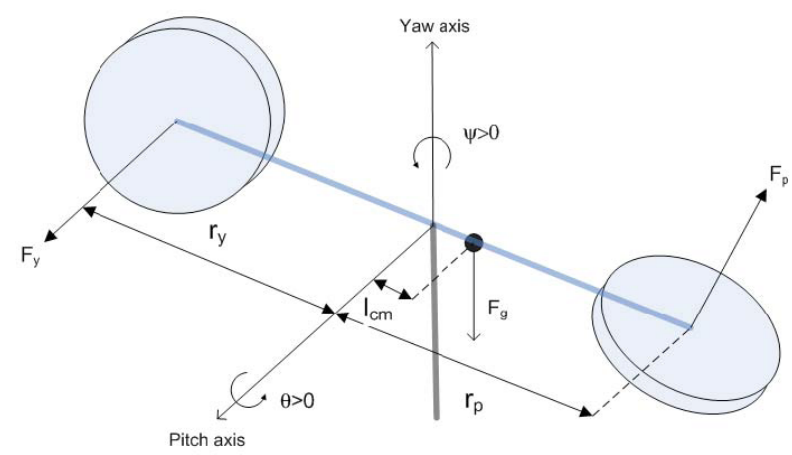

Figura. 2. Dinámica del helicóptero experimental Quanser.

$$
\begin{aligned}
& \left(\boldsymbol{J}_{e q, p}+\boldsymbol{m}_{\text {heli }} \boldsymbol{l}_{c m}^{2}\right) \ddot{\boldsymbol{\theta}}=\boldsymbol{K}_{p p} \boldsymbol{V}_{\boldsymbol{m}, \boldsymbol{p}}+\boldsymbol{K}_{p y} \boldsymbol{V}_{m, y}-\boldsymbol{m}_{\text {heli }} \boldsymbol{g} \boldsymbol{l}_{c m} \cos \boldsymbol{\theta} \\
& -\boldsymbol{B}_{p} \dot{\boldsymbol{\theta}}-\boldsymbol{m}_{\text {heli }} \boldsymbol{l}_{c m}^{2} \sin \boldsymbol{\theta} \cos \theta \dot{\psi}^{2} \\
& \left(\boldsymbol{J}_{e q, y}+\boldsymbol{m}_{\text {heli }} \boldsymbol{l}_{c m}^{2} \cos \boldsymbol{\theta}\right) \ddot{\psi}=\boldsymbol{K}_{y y} \boldsymbol{V}_{m, y}+\boldsymbol{K}_{y p} \boldsymbol{V}_{m, p}-\boldsymbol{B}_{y} \dot{\psi} \\
& +2 \boldsymbol{m}_{\text {heli }} \boldsymbol{l}_{c m}^{2} \sin \boldsymbol{\theta} \cos \boldsymbol{\theta} \dot{\boldsymbol{\theta}} \dot{\boldsymbol{\theta}}
\end{aligned}
$$

De las ecuaciones no lineales de movimientos (1) se obtiene el modelo de espacio de estados lineal del helicóptero, sobre el punto de operación $\mathrm{X}_{0}=(0,0,0,0)$, obteniendo (2).

$$
\begin{aligned}
& \left(J_{e q, p}+\boldsymbol{m}_{h e l i} l_{c m}^{2}\right) \ddot{\theta}=K_{p p} V_{m, p}+K_{p y} V_{m, y}-B_{p} \dot{\theta} \\
& \left(J_{e q, y}+\boldsymbol{m}_{h e l i} l_{c m}^{2}\right) \ddot{\psi}=K_{y y} V_{m, y}+K_{y p} V_{m, p}-B_{y} \dot{\psi}
\end{aligned}
$$

Con: $\boldsymbol{x}=\left[\begin{array}{llll}\boldsymbol{\theta} & \psi & \dot{\theta} & \dot{\psi}\end{array}\right]^{T} \mathrm{y} \quad \boldsymbol{u}=\left[\begin{array}{lll}\boldsymbol{V}_{m, p} & \boldsymbol{V}_{m, y}\end{array}\right]^{T}$, se obtiene el modelo nominal (3), los valores dados por el fabricante del helicóptero se pueden observar en la Tabla 1.

Al realizar el análisis de controlabilidad, estabilidad y observabilidad del sistema nominal se observa que la matriz de controlabilidad del sistema tiene rango 4, al igual que la matriz de observabilidad tiene rango 4 y los valores propios del sistema son $[0,0,-9.2751,-3.4955]$. 
Lo que demuestra que el sistema es marginalmente inestable, ya que posee dos valores propios en 0 .

Tabla 1. Valores nominales del sistema Quanser 2DOF.

\begin{tabular}{|c|c|c|}
\hline Símbolo & Descripción & Valor \\
\hline Крр & $\begin{array}{l}\text { Thrust torque constant acting } \\
\text { on Pitch axis from Pitch motor/ } \\
\text { propeller }\end{array}$ & 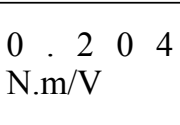 \\
\hline Куу & $\begin{array}{l}\text { Thrust torque constant acting } \\
\text { on Yaw axis from Yaw motor/ } \\
\text { propeller }\end{array}$ & $\begin{array}{l}0.0772 \\
\text { N.m/V }\end{array}$ \\
\hline Кру & $\begin{array}{l}\text { Thrust torque constant acting } \\
\text { on Pitch axis from Yaw motor/ } \\
\text { propeller. }\end{array}$ & $\begin{array}{l}0.00668 \\
\text { N.m/V }\end{array}$ \\
\hline Кур & $\begin{array}{l}\text { Thrust torque constant acting } \\
\text { on Yaw axis from Pitch motor/ } \\
\text { propeller }\end{array}$ & 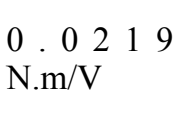 \\
\hline Bp & $\begin{array}{l}\text { Equivalent viscous damping } \\
\text { about Pitch axis }\end{array}$ & $0.800 \mathrm{~N} / \mathrm{V}$ \\
\hline By & $\begin{array}{l}\text { Equivalent viscous damping } \\
\text { about Yaw axis }\end{array}$ & $0.318 \mathrm{~N} / \mathrm{V}$ \\
\hline $\boldsymbol{m}_{\text {heli }}$ & $\begin{array}{l}\text { Total moving mass of the } \\
\text { helicopter. } 1.3872 \mathrm{~kg} \mathrm{lcm} 1 \mathrm{~cm} \\
\text { Center-of-mass length along } \\
\text { helicopter body from Pitch axis }\end{array}$ & $1.3872 \mathrm{~kg}$ \\
\hline lcm & $\begin{array}{l}\text { Center-of-mass length along } \\
\text { helicopter body from Pitch axis }\end{array}$ & $0.186 \mathrm{~cm}$ \\
\hline Jeq,p & $\begin{array}{l}\text { Total moment of inertia about } \\
\text { Pitch pivot }\end{array}$ & $\begin{array}{l}0.0384 \mathrm{~kg} \\
\mathrm{~m}^{2}\end{array}$ \\
\hline Jeq,y & $\begin{array}{l}\text { Total moment of inertia about } \\
\text { Yaw pivot }\end{array}$ & $\begin{array}{l}0.0432 \mathrm{~kg} \\
\mathrm{~m}^{2}\end{array}$ \\
\hline
\end{tabular}

Fuente: Quanser 2 DOF Helicopter User and Control Manual.

$\dot{X}=\left[\begin{array}{cccc}0 & 0 & 1 & 0 \\ 0 & 0 & 0 & 1 \\ 0 & 0 & -\frac{B_{p}}{J_{e q, p}+m_{h e l i} l_{c m}^{2}} & 0 \\ 0 & 0 & 0 & -\frac{B_{y}}{J_{e q, y}+m_{h e l i} l_{c m}^{2}}\end{array}\right] X$

$+\left[\begin{array}{cc}0 & 0 \\ 0 & 0 \\ \frac{K_{p p}}{J_{e q, p}+m_{h e l i} l_{c m}^{2}} & \frac{K_{p y}}{J_{e q, p}+m_{h e l i} l_{c m}^{2}} \\ \frac{K_{y p}}{J_{e q, y}+m_{h e l i} l_{c m}^{2}} & \frac{K_{y y}}{J_{e q, y}+m_{h e l i} l_{c m}^{2}}\end{array}\right] U$

$Y=\left[\begin{array}{llll}1 & 0 & 0 & 0 \\ 0 & 1 & 0 & 0 \\ 0 & 0 & 1 & 0 \\ 0 & 0 & 0 & 1\end{array}\right] X+\left[\begin{array}{ll}0 & 0 \\ 0 & 0 \\ 0 & 0 \\ 0 & 0\end{array}\right] U$

\section{DISEÑO DEL CONTROLADOR}

\subsection{Especificaciones de desempeño}

En cuanto a las consideraciones de desempeño, se requiere que en estado estacionario las posiciones Pitch y Yaw se mantengan en $\mathrm{X}_{0}$ dado, $\mathrm{y}$ se rechacen pequeñas perturbaciones; es decir, se desea error de estado estacionario nulo. Se tiene ruido de medición de la posición $\theta$ y $\psi$, por tanto hay que diseñar un filtro en la entrada de medición, de tal manera que rechace las señales de entrada de alta frecuencia. La acción de control debe ser penalizada en alta frecuencia con alguna función de peso, ya que se tiene una relación directa de la señal de control y la respuesta del sistema. Adicionalmente, la velocidad angular, tanto de Pitch como de Yaw, no puede ser medida, por lo que se debe diseñar un filtro que nos proporcione dichas señales para el controlador. Se deben evitar picos excesivos en los voltajes de los motores, para evitar daños de tipo físico, por tanto, los actuadores tienen señales máximas, de $\pm 24 \mathrm{~V}$ para el motor de Pitch y de $\pm 15 \mathrm{~V}$ para el motor de Yaw, por lo que se deben implementar saturadores en el sistema.

\subsection{Esquema general del sistema}

Para el diseño del controlador robusto $\mathrm{H} \infty$ basado en técnicas LMI, se tiene en cuenta el modelo general dado en (4), el cual corresponde al modelo de la planta $\mathrm{P}(\mathrm{s})$ del sistema realimentado presente en la Figura 3.

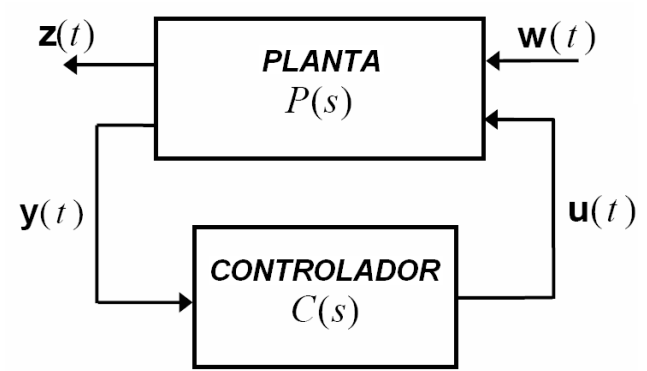

Figura. 3. Interconexión por realimentación de la planta y el controlador.

$$
\begin{aligned}
& \dot{X}(t)=A X(t)+B_{1} w(t)+B_{2} u(t) \\
& Z=C_{1} X(t)+D_{11} w(t)+D_{12} u(t) \\
& Y=C_{2} X(t)+D_{21} w(t)+D_{22} u(t)
\end{aligned}
$$




\subsection{Modelo con incertidumbres}

Las incertidumbres se plantean como una variación del sistema nominal de la matriz A y la matriz B; es decir, se plantea un sistema como el presente en (5), para de esta forma llegar a (6). Primero, para la variación de los parámetros, se hizo la variación en un $\pm 10 \%$ sobre el valor nominal. Los parámetros que se variaron fueron $\mathrm{J}_{\text {eq.p }}, \mathrm{J}_{\text {eq.y }}, \mathrm{B}_{\mathrm{p}}, \mathrm{B}_{\mathrm{y}}, \mathrm{K}_{\mathrm{pp}}, \mathrm{K}_{\mathrm{yy}}, \mathrm{K}_{\mathrm{yp}}, \mathrm{y} \mathrm{K}_{\mathrm{py}}$, y las variaciones sobre el punto de operación se realizaron para el ángulo de Pitch y de Yaw de \pm 15 grados, y debido a que se desea que las velocidades angulares sean cero, la variación sobre estos se hizo de \pm 10 grados.

$$
\begin{aligned}
& \dot{\boldsymbol{X}}=\left(\boldsymbol{A}+\Delta_{A}\right) \boldsymbol{X}+\left(\boldsymbol{B}+\Delta_{\boldsymbol{B}}\right) \boldsymbol{U} \\
& \boldsymbol{Y}=\boldsymbol{C} \boldsymbol{X}+\boldsymbol{D} \boldsymbol{U} \\
& \dot{\boldsymbol{X}}=\boldsymbol{A} \boldsymbol{X}+\left(\Delta_{A} \boldsymbol{X}+\Delta_{\boldsymbol{B}} \boldsymbol{U}\right)+\boldsymbol{B} \boldsymbol{U}
\end{aligned}
$$

Después de realizar las variaciones se logra encontrar que las variables que más influyen en la incertidumbre son los elementos de las filas 3 y 4 de las columnas 3 y 4 de la matriz A y las dos columnas de la matriz B. Con base en esto se obtiene la matriz $\mathrm{B}_{1}$, la cual estaría descrita en (7).

$$
\boldsymbol{B}_{1}=\left[\begin{array}{cccc}
0 & 0 & 0 & 0 \\
0 & 0 & 0 & 0 \\
0.3571 & 0.0120 & 0.4311 & 0.0432 \\
0.0372 & 0.123 & 0.0418 & 0.5427
\end{array}\right] \mapsto \boldsymbol{W}\left[\begin{array}{l}
\boldsymbol{w}_{1} \\
\boldsymbol{w}_{2} \\
\boldsymbol{w}_{3} \\
\boldsymbol{w}_{4}
\end{array}\right]
$$

\subsection{Diseño del controlador LMI}

El diseño del controlador LMI está basado en el sistema (4), considerando la señal de control como en (8), se obtiene el sistema presentado en (9).

$$
u(t)=-K X(t)
$$

$$
\begin{aligned}
& \dot{X}(\boldsymbol{t})=\underbrace{\left(A-B_{2} K\right)}_{\text {Alc }} X(t)+B_{1} w(t) \\
& Z=\underbrace{\left(C_{1}-D_{12} K\right)}_{\text {Clc }} X(t)+D_{11} w(t) \\
& Y=\left(C_{2}-D_{22} K\right) X(t)+D_{21} w(t) \\
& Z(t)=\left[C l c(S I-A l c)^{-1} B_{1}+D_{11}\right] X(t)
\end{aligned}
$$

Con la función del sistema en lazo cerrado (10), se diseña un controlador por realimentación de estados empleando técnicas LMI, entonces, considerando $\mathrm{C}_{2}=\mathrm{I}^{\mathrm{nxn}}$ y $\mathrm{D}_{21}=0$. El problema es encontrar la matriz de realimentación de estados óptima $\mathrm{H} \infty, \mathrm{K} \in \mathfrak{R}^{2 \times n}$, tal que $\| \mathrm{FL}(\mathrm{P}(\mathrm{s}), \mathrm{K})$ $\|_{\infty}$ sea mínima, para esto enunciamos el siguiente lema (Kebede, 2006).

LEMA (Bounded Real Lemma for Continuous-Time Systems): considere una función de transferencia continua $T(s)$, la cual no necesariamente es de realización mínima, $T(s)=\mathbf{C}(s \mathbf{I}-\mathbf{A})^{-1} \mathbf{B}+\mathbf{D}$. Entonces, $T(s)<\gamma$, para $\gamma>0$ y A será asintóticamente estable si y solo si existe una matriz simétrica definida positiva, $\mathbf{P}>\mathbf{0}$, que satisface la siguiente LMI:

$$
\left[\begin{array}{ccc}
P A+A^{T} P & P B & C^{T} \\
B^{T} P & -\gamma I & D^{T} \\
C & D & -\gamma I
\end{array}\right]<0
$$

Aplicando el lema anterior al sistema en lazo cerrado se obtiene (12); empleando las propiedades dadas en (13), se puede llegar finalmente a que el diseño del controlador se da como un problema de optimización.

$$
\left[\begin{array}{ccc}
\boldsymbol{P}\left(\boldsymbol{A}-\boldsymbol{B}_{2} \boldsymbol{K}\right)+\left(\boldsymbol{A}-\boldsymbol{B}_{2} \boldsymbol{K}\right)^{T} \boldsymbol{P} & \boldsymbol{P B}_{1} & \left(\boldsymbol{C}_{1}-\boldsymbol{D}_{12} \boldsymbol{K}\right)^{T} \\
\boldsymbol{B}_{1}{ }^{T} \boldsymbol{P} & -\boldsymbol{\gamma I} & \boldsymbol{D}_{11}{ }^{T} \\
\left(\boldsymbol{C}_{1}-\boldsymbol{D}_{12} \boldsymbol{K}\right) & \boldsymbol{D}_{11} & -\boldsymbol{\gamma} \boldsymbol{I}
\end{array}\right]<0
$$

$$
\begin{aligned}
& \boldsymbol{L}=\boldsymbol{K} \boldsymbol{Q} \mapsto \boldsymbol{K}=\boldsymbol{L} \boldsymbol{Q}^{-1} \mapsto \boldsymbol{Q}=\boldsymbol{P}^{-1}>0 \\
& \boldsymbol{P}=\boldsymbol{P}^{\boldsymbol{T}}>0 \\
& \boldsymbol{Y}=\boldsymbol{P} \boldsymbol{X} \in \mathfrak{R}^{n} \rightarrow \boldsymbol{X}=\boldsymbol{P}^{-1} \boldsymbol{Y} \rightarrow \boldsymbol{X}^{\boldsymbol{T}}=\boldsymbol{Y}^{\boldsymbol{T}} \boldsymbol{P}^{-1}
\end{aligned}
$$


El problema de estabilidad de la síntesis del control optimo $\left(\boldsymbol{K}=\boldsymbol{L} \boldsymbol{Q}^{-1}\right)$ por realimentación - Ho es minimizar $\gamma \in \Re$ con $\boldsymbol{Q}>\mathbf{0}$, tal que la siguiente LMI sea factible.

$$
\left[\begin{array}{ccc}
\boldsymbol{A} \boldsymbol{Q}+\boldsymbol{Q} \boldsymbol{A}^{\boldsymbol{T}}-\left(\boldsymbol{B}_{2} \boldsymbol{L}+\boldsymbol{L}^{T} \boldsymbol{B}_{2}{ }^{T}\right) & \boldsymbol{B}_{1} & \left(\boldsymbol{C}_{1} \boldsymbol{Q}-\boldsymbol{D}_{12} \boldsymbol{L}\right)^{\boldsymbol{T}} \\
\boldsymbol{B}_{1} & -\boldsymbol{\gamma I} & \boldsymbol{D}_{11}{ }^{T} \\
\boldsymbol{C}_{1} \boldsymbol{Q}-\boldsymbol{D}_{12} \boldsymbol{L} & \boldsymbol{D}_{11} & -\boldsymbol{\gamma} \boldsymbol{I}
\end{array}\right]<0
$$

La optimización de este problema para lograr el mínimo valor de $\gamma$ se realiza empleando el Toolbox de LMI de Matlab, obteniendo como resultado para el sistema nominal y la matriz $\mathrm{B}_{1}$ definidas anteriormente:

$$
\begin{aligned}
\boldsymbol{\gamma} & =\mathbf{0 . 3 7} \\
\boldsymbol{K} & =\left[\begin{array}{cccc}
264.6983 & 21.7836 & 21.3092 & 1.7475 \\
-41.2847 & 5.1074 & -3.4622 & 2.4247
\end{array}\right]
\end{aligned}
$$

Valores singulares de Alc $=[-46.0565-13.5920-1.5195$ -3.8832], con este controlador $\mathbf{K}$ se procede a realizar la simulación y el análisis de robustez del sistema en lazo cerrado.

\subsubsection{Consideraciones para la simulación.}

Debido a que la velocidad angular, tanto de Pitch como de Yaw, no puede ser medida, se utiliza un filtro para determinar estas magnitudes, tanto para el cálculo de la velocidad angular de Pitch como de Yaw. Es un filtro de segundo orden con los siguientes parámetros: frecuencia de corte Wc de $21 \mathrm{rad} / \mathrm{sec}$ y coeficiente de amortiguamiento de 0.75 , obteniendo la función de transferencia (15).

$$
V_{f}(s)=\frac{441 s}{s^{2}+31.5 s+441} x(s)
$$

Adicionalmente, la posición Pitch es regulada utilizando un lazo de realimentación feed-forward que compensa el torque gravitacional $\left(\boldsymbol{\tau}_{\mathrm{g}}\right)$, la señal de control feed-forward (FF) está dada por (16), donde $\theta_{d}$ es el ángulo de Pitch deseado y $\mathrm{K}_{\mathrm{ff}}$ es la ganancia de control feed-forward.

$\boldsymbol{u}_{f f}=K_{f f} \frac{\boldsymbol{m}_{h e l i} g \boldsymbol{l}_{c m} \cos \theta_{d}}{K p p}$

En resumen, el control FF+LMI está dado por (17), donde los valores de la constante $\mathrm{K}$ son los hallados anteriormente.

$$
\left[\begin{array}{c}
\boldsymbol{u}_{\text {pitch }} \\
\boldsymbol{u}_{\text {yaw }}
\end{array}\right]=\left[\begin{array}{c}
\boldsymbol{K}_{f f} \frac{\boldsymbol{m}_{\text {heli }} \boldsymbol{g l} \boldsymbol{l}_{c m} \cos \theta_{d}}{\boldsymbol{K p p}} \\
0
\end{array}\right]-K\left[\begin{array}{c}
\theta-\theta_{d} \\
\psi-\psi_{d} \\
\dot{\theta} \\
\dot{\psi}
\end{array}\right]
$$

Donde $\theta_{d}$ es el ángulo de Pitch deseado y $\psi_{d}$ es el ángulo de Yaw deseado, y el valor de la ganancia de control feed-forward $\mathrm{K}_{\mathrm{ff}}$ es 1 .

\subsubsection{Simulación control FF+ LMI}

Con los valores del controlador ya determinados se procede a realizar la simulación con el modelo no lineal del sistema. Primero se hace la simulación del sistema sin emplear el filtro de cálculo de velocidad angular y posteriormente con dicho filtro, obteniendo los resultados mostrados en las Figuras 4 y 5 . 


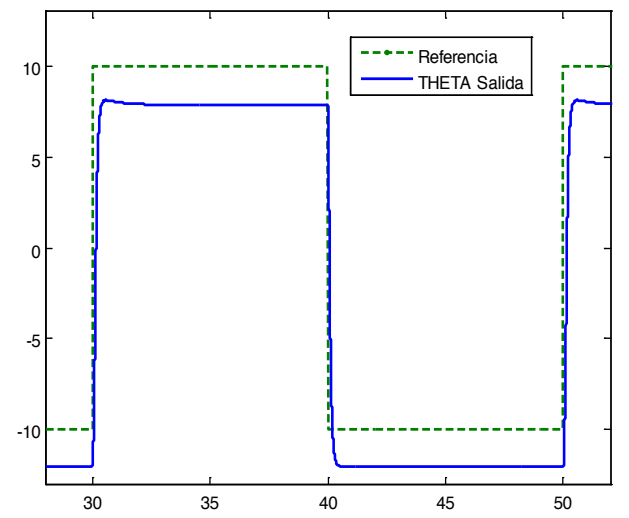

a) Angulo de Pitch

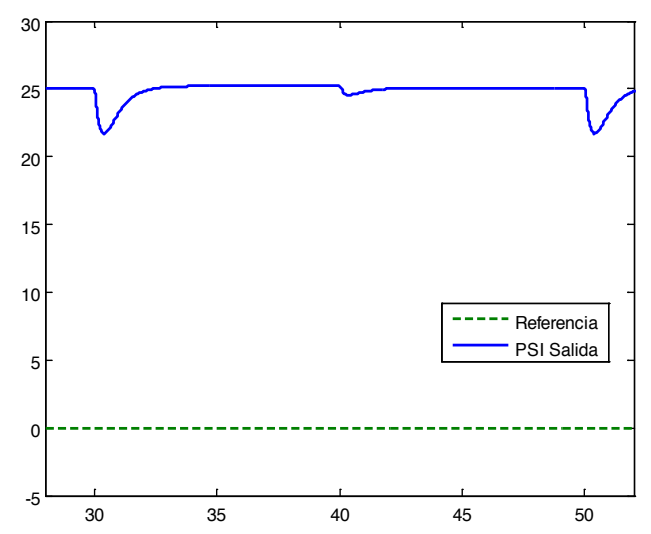

b) Angulo de Yaw.

Figura 4. Respuesta del sistema al controlador FF + LMI. a) Angulo de Pitch. b) Angulo de Yaw.

Como se observa de las Figuras 4 y 5 , el error de posición en estado estacionario del ángulo de Yaw es bastante elevado. Además, cuando se utiliza el filtro para determinar las velocidades angulares, se causa una oscilación en la posición del ángulo de Pitch así como en el ángulo de Yaw, aunque en menor medida. Es necesario entonces diseñar un controlador que minimice tanto el error como la oscilación, por lo que se lleva a cabo el diseño de un controlador FF+LMI con integrador.

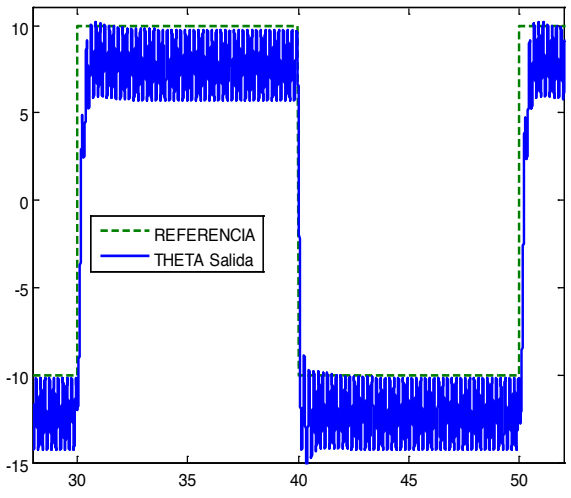

a) Ángulo de Pitch

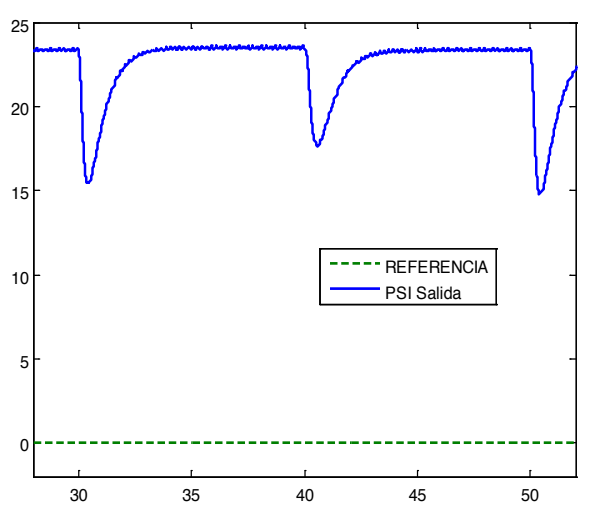

b) Angulo de Yaw.

Figura 5. Respuesta del sistema al controlador FF + LMI con filtros para determinar las velocidades angulares. a) Ángulo de Pitch. b) Angulo de Yaw.

\subsection{Diseño del controlador FF+LMI con integrador}

Para minimizar el error de posición en estado estacionario según las características deseadas, se introduce un integrador al control LMI. Al establecer los estados $X_{5}=$ $\theta$ y $X_{6}=\psi$, se obtiene el sistema nominal (18) y la matriz $B_{1}$ dada en (19). 
Con estos nuevos valores de las matrices se realiza el cálculo del controlador $\mathrm{K} \in \Re^{2 \times 6}$, minimizando igual que en el caso anterior el valor de $\gamma \in \Re$ con $\boldsymbol{Q}>\boldsymbol{0}$, tal que la LMI (14) sea factible.

$$
\begin{aligned}
\dot{\boldsymbol{X}} & =\left[\begin{array}{cccccc}
0 & 0 & 1 & 0 & 0 & 0 \\
0 & 0 & 0 & 1 & 0 & 0 \\
0 & 0 & -9.2751 & 0 & 0 & 0 \\
0 & 0 & 0 & -3.4955 & 0 & 0 \\
1 & 0 & 0 & 0 & 0 & 0 \\
0 & 1 & 0 & 0 & 0 & 0
\end{array}\right] \boldsymbol{X}+\left[\begin{array}{ccc}
0 & 0 \\
0 & 0 \\
2.3667 & 0.0790 \\
0.2410 & 0.7913 \\
0 & 0 \\
0 & 0
\end{array}\right] \boldsymbol{U} \\
\boldsymbol{Y} & =\left[\begin{array}{llllll}
1 & 0 & 0 & 0 & 0 & 0 \\
0 & 1 & 0 & 0 & 0 & 0 \\
0 & 0 & 1 & 0 & 0 & 0 \\
0 & 0 & 0 & 1 & 0 & 0 \\
0 & 0 & 0 & 0 & 1 & 0 \\
0 & 0 & 0 & 0 & 0 & 1
\end{array}\right] \boldsymbol{X}
\end{aligned}
$$

$$
\boldsymbol{B}_{1}=\left[\begin{array}{cccccc}
0 & 0 & 0 & 0 & 0 & 0 \\
0 & 0 & 0 & 0 & 0 & 0 \\
0.3571 & 0.0120 & 0.4311 & 0.0432 & 0 & 0 \\
0.0372 & 0.123 & 0.0418 & 0.5427 & 0 & 0 \\
0 & 0 & 0 & 0 & 0 & 0 \\
0 & 0 & 0 & 0 & 0 & 0
\end{array}\right] \mapsto \boldsymbol{W}\left[\begin{array}{c}
\boldsymbol{w}_{1} \\
\boldsymbol{w}_{2} \\
\boldsymbol{w}_{3} \\
\boldsymbol{w}_{4} \\
\boldsymbol{w}_{5} \\
\boldsymbol{w}_{6}
\end{array}\right]
$$

Como resultado de este cálculo se obtiene:

$\boldsymbol{\gamma}=0.5$
$\boldsymbol{K} \boldsymbol{i}=\left[\begin{array}{cccccc}33.7221 & 3.0898 & 2.8430 & 0.0150 & 16.3401 & 1.1710 \\ -6.4109 & 15.7772 & -0.7629 & 3.9787 & -3.5094 & 9.5534\end{array}\right]$

Valores singulares de Alc $=[-7.6384+3.4219 \mathrm{i}-7.6384$ - $3.4219 \mathrm{i}-3.9249-1.2465+0.4723 \mathrm{i}-1.2465-0.4723 \mathrm{i}$ -0.5381 ], con este controlador $\mathbf{K i}$ se procede a realizar la simulación y el análisis de robustez del sistema en lazo cerrado. Además, la parte integral se implementa con un control integral anti-windup como el presentado en el manual de Quanser 2 DOF.

Este control integral anti-windup se emplea tanto para el ángulo de Pitch como de Yaw. Los resultados de la simulación con el filtro de cálculo de velocidad angular se pueden observar en las Figuras 6 a 8 . En la Figura 8 se puede observar la respuesta ante perturbaciones de tipo escalón que ocasionan cambios en la señal de salida de $\pm 5^{\circ}$. Como se aprecia, el sistema es capaz de rechazar pequeñas perturbaciones de tipo escalón en la salida y el error tiende a ser cero.

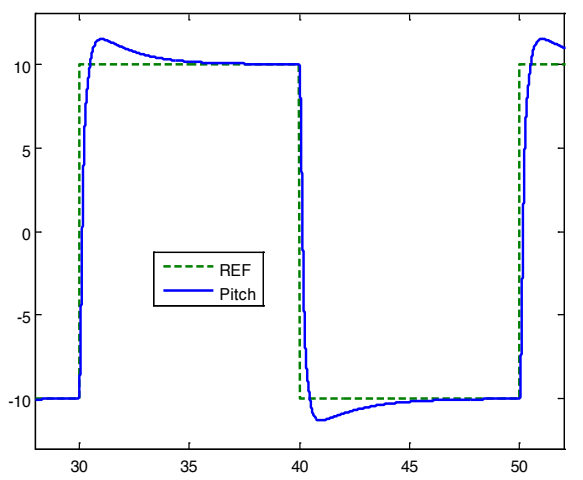

a) Ángulo de Pitch

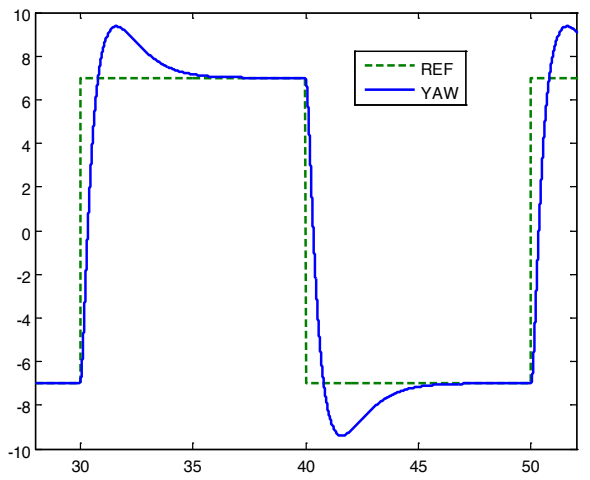

b) Angulo de Yaw.

Figura. 6. Respuesta del sistema al controlador FF+LMI+Integrador con filtros para determinar las velocidades angulares. a) Ángulo de Pitch. b) Angulo de Yaw. 


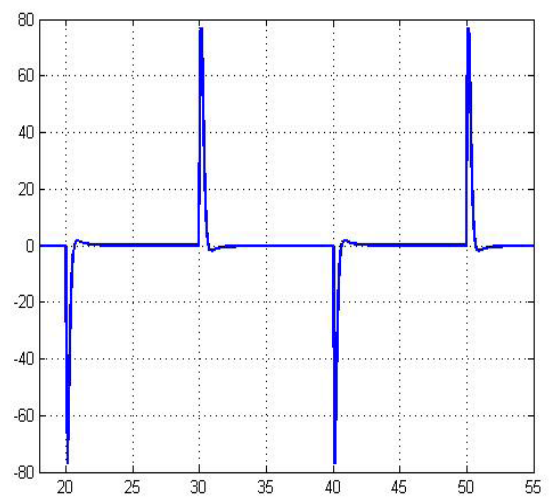

a) Velocidad angular de Pitch

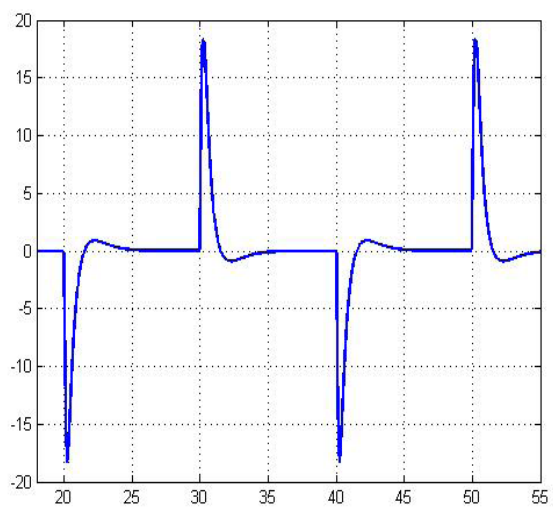

b) Velocidad angular de Yaw.

Figura.7.Respuesta del sistema al controlador FF+LMI+ Integrador con filtros para determinar las velocidades angulares. a) Velocidad angular de Pitch. b) Velocidad angular de Yaw.

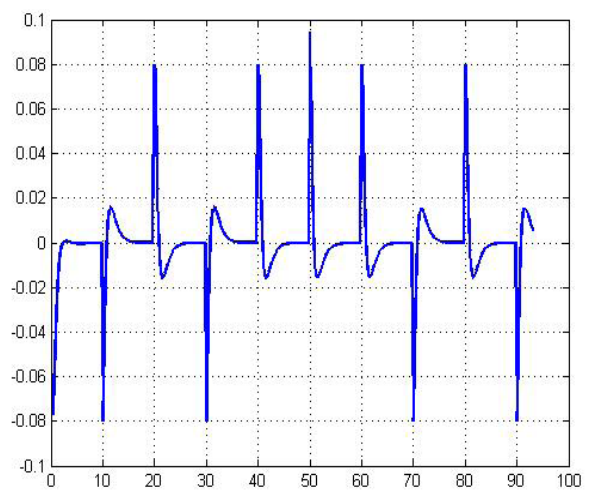

a) Error ángulo de Pitch

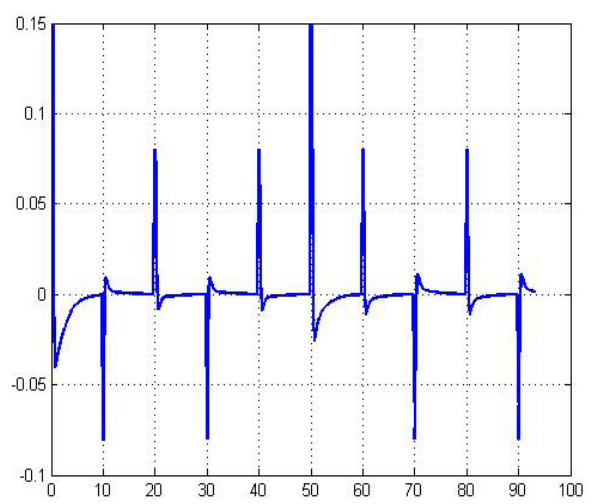

b) Error ángulo de Yaw.

Figura. 8. Respuesta del sistema a perturbaciones, controlador LMI+Integrador con filtros para las velocidades angulares. a) Error ángulo de Pitch. b) Error ángulo de Yaw 


\section{ESTABILIDAD ROBUSTA}

Considerando el diseño por LMI y LMI+I realizados anteriormente, la gráfica de estabilidad robusta para el controlador LMI se observa en la Figura 9 y para el controlador LMI+I se aprecia en la Figura 10. En estas figuras se nota que el sistema controlado alcanza su estabilidad robusta debido a que los valores singulares de la función de sensibilidad ponderada de entrada tienen un valor pico de 0.11 aproximadamente. La estabilidad robusta del sistema Quanser 2 DOF con los controladores diseñados se realiza utilizando $\mu$-análisis.

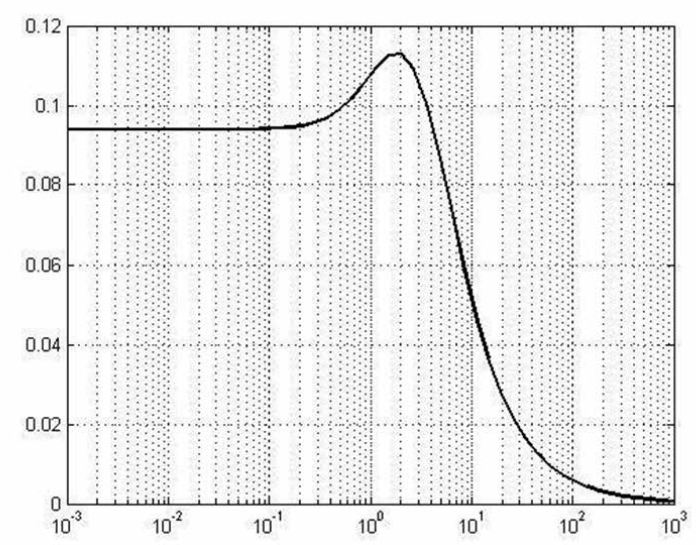

Figura. 9. Estabilidad robusta del sistema al controlador LMI

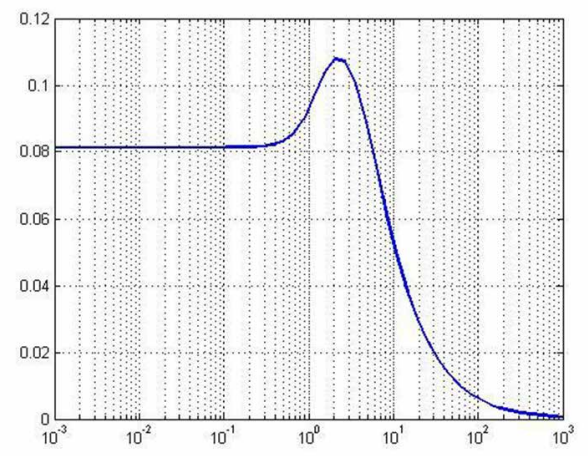

Figura. 10. Estabilidad robusta del sistema al controlador LMI+ Integrador

\section{COMPARACIÓN ENTRE CONTROLADORES}

Se efectúa la comparación de los desempeños de los diseños realizados con los presentados por el fabricante del dispositivo en su manual. Las condiciones iniciales del sistema son $(0,0,0,0)$. Los resultados de la comparación entre el controlador $\mathrm{FF}+\mathrm{LQR}$ y FF+LMI son los mostrados en las Figuras 11 y 12. De estas figuras se logra observar que aunque el error en el ángulo de Yaw es bastante elevado y se presenta un error menor en el ángulo de Pitch (7-8 \% para el control LMI y de 25 $\%$ para el control LQR), el controlador FF+LMI obtiene el menor error entre los dos controladores $\mathrm{FF}+\mathrm{LQR}$ y FF+LMI.

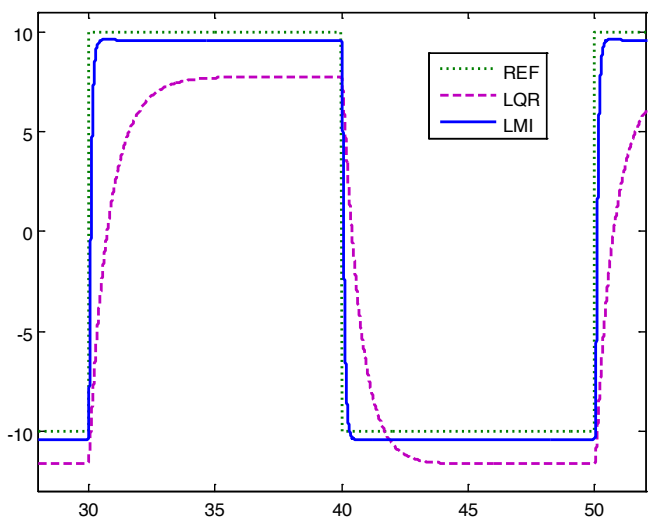

Figura. 11. Respuesta del sistema a los controladores FF+LQR y FF+LMI (ángulo de Pitch)

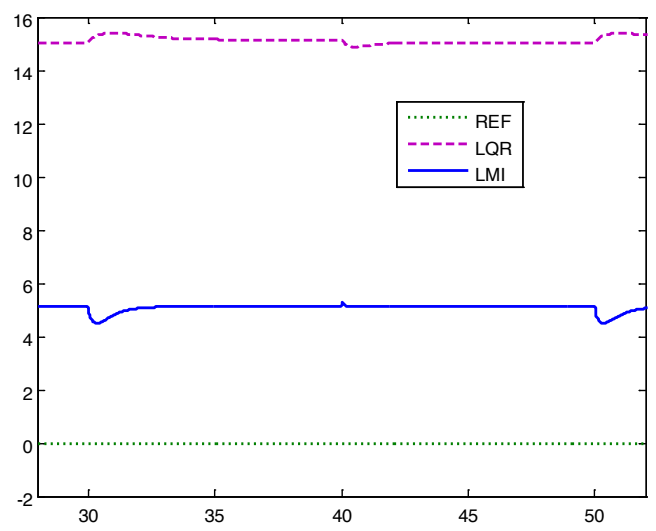

Figura. 12. Respuesta del sistema a los controladores FF+LQR y FF+LMI (ángulo de Yaw)

Los resultados de la comparación entre el controlador $\mathrm{FF}+\mathrm{LQR}+\mathrm{I}$ y $\mathrm{FF}+\mathrm{LMI}+$ Integrador son los presentados en las Figuras 13 y 14. De estas figuras se logra observar para los dos casos que el error en el ángulo de Yaw y en el ángulo de Pitch es cero; el controlador FF+LMI+I obtiene el menor tiempo de establecimiento tanto para el 
ángulo de Pitch como de Yaw, con un sobrepico para el control FF+LMI+I del $20 \%$ para el ángulo de Pitch y de $30 \%$ para el ángulo de Yaw, y sobrepico para el control $\mathrm{FF}+\mathrm{LQR}+\mathrm{I}$ del $30 \%$ para el ángulo de Pitch y de $30 \%$ para el ángulo de Yaw.

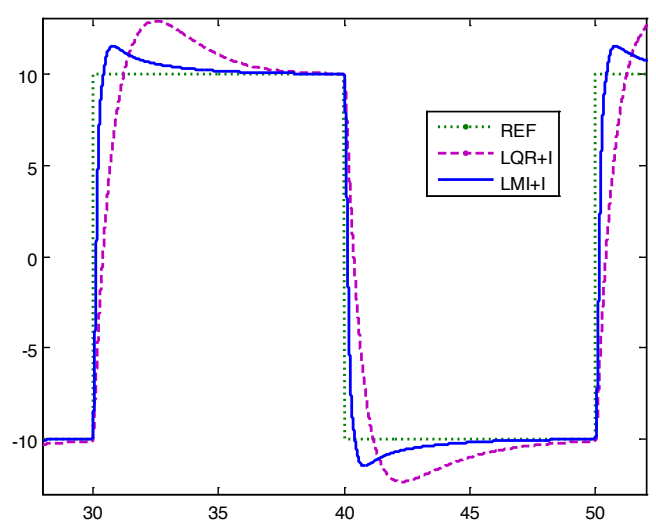

Figura. 13. Respuesta del sistema a los controladores FF+L$\mathrm{QR}+\mathrm{I}$ y FF+LMI+I (ángulo de Pitch)

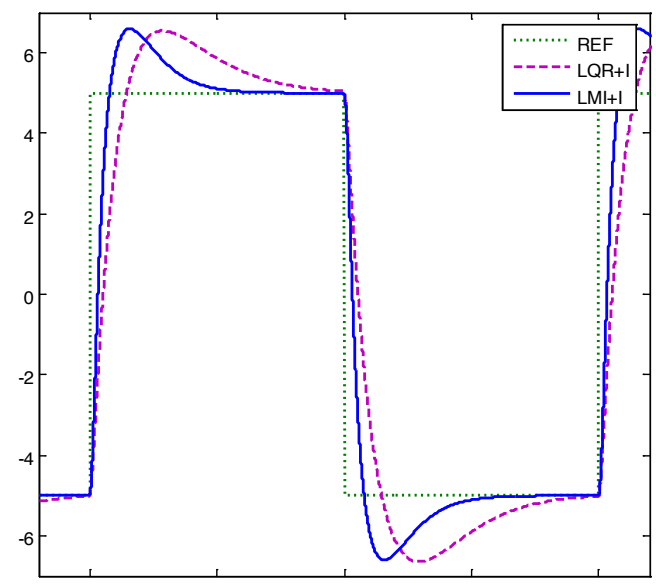

Figura. 14. Respuesta del sistema a los controladores FF+L$\mathrm{QR}+\mathrm{I}$ y FF+LMI+I (ángulo de Yaw).

Por último se hace una prueba para determinar el comportamiento del control $\mathrm{FF}+\mathrm{LQR}+\mathrm{I}$ y $\mathrm{FF}+\mathrm{LMI}+\mathrm{I}$ (son los que mejores resultados han tenido) ante el seguimiento a una señal tipo diente de sierra para el ángulo Pitch (frecuencia de 0.05 Hertz) y una señal tipo escalón para el ángulo de Yaw (frecuencia 0.04 Hertz) (Figuras 15 y 16). Se observa que el seguimiento es realizado por los dos sistemas controlados, en el ángulo de Pitch el error en el punto donde cambia abruptamente el diente de sierra es menor para el controlador $\mathrm{FF}+\mathrm{LMI}+\mathrm{I}$, pero este presenta una oscilación en el punto donde cambia la referencia del ángulo de Yaw, mientras que el control $\mathrm{FF}+\mathrm{LQR}+\mathrm{I}$ no presenta dicha oscilación. Algo similar ocurre en el ángulo de Yaw, donde se produce la oscilación en el punto de cambio abrupto del diente de sierra, siendo menor la oscilación en el controlador $\mathrm{FF}+\mathrm{LQR}+\mathrm{I}$.

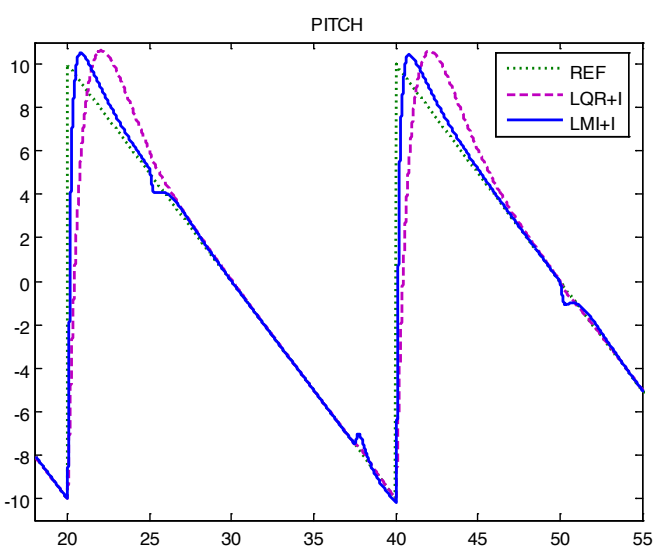

Figura. 15. Respuesta del sistema al controlador $\mathrm{FF}+\mathrm{LQR}+\mathrm{I}$ y FF+LMI+I (ángulo de Pitch)

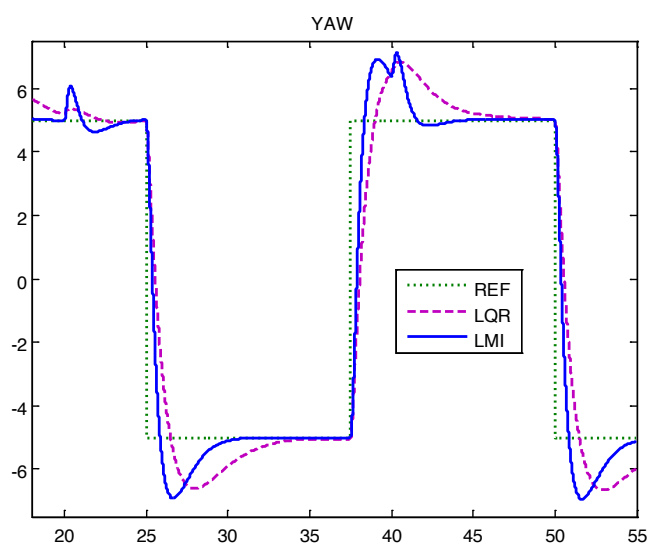

Figura. 16. Respuesta del sistema al controlador $\mathrm{FF}+\mathrm{LQR}+\mathrm{I}$ y $\mathrm{FF}+\mathrm{LMI}+\mathrm{I}$ (ángulo de Yaw)

\section{CONCLUSIONES}

Se puede concluir que la dinámica del controlador $\mathrm{FF}+\mathrm{LMI}+\mathrm{I}$ es superior a la presentada por el controlador $\mathrm{FF}+\mathrm{LQR}+\mathrm{I}$, su principal inconveniente es que la exigencia de velocidad angular es mucho más elevada, 
cerca del $200 \%$ con respecto a la exigida por el control $\mathrm{FF}+\mathrm{LQR}+\mathrm{I}$.

Las diferencias observadas en los controladores diseñados frente a los controladores dados por el fabricante radican en los tiempos de establecimiento, y error en estado estacionario. El controlador LMI, aunque presentaba un desempeño mejor que el control LQR, mostraba un error elevado en el seguimiento al ángulo de Yaw, por lo que se descarta su utilización, mientras que el controlador $\mathrm{FF}+\mathrm{LMI}+\mathrm{I}$ mostró un excelente comportamiento con un tiempo de establecimiento inferior a los 5 segundos y sobrepicos de $20 \%$ para el seguimiento en el ángulo de Pitch y de $30 \%$ en el ángulo de Yaw, menores que los presentados por el controlador $\mathrm{FF}+\mathrm{LQR}+\mathrm{I}$. Por consiguiente, se concluye que el controlador más robusto frente a perturbaciones, que cumple con las características deseadas y presenta un error de seguimiento cero, es el controlador FF+LMI+I.

\section{REFERENCIAS}

Balas, G. \& Doyle, J. (2003). $\mu$-Analysis and Synthesis Toolbox User's Guide Version 3 for use to MAT$L A B$. Massachusetts, USA. Math Works Inc.

Banjerdpongchai, D. (1997) Parametric Robust Controller Synthesis Using Linear Matrix Inequalities. Thesis to get Phd. in Philosophy. Stanford University, California.

Bedoya, A. J. \& Marín, A. A. (2014). Construcción y control adaptativo de un helicóptero de dos grados de libertad. Trabajo de grado para optar al título de ingeniero en mecatrónica Universidad Tecnológica de Pereira.

Carrillo-Ahumada, J., Reynoso-Meza, G., García-Nieto, S., Sanchis, J. \& García-Alvarado, M. A. (2015). Sintonización de controladores Pareto-óptimo robustos para sistemas multivariables. Aplicación en un helicóptero de 2 grados de libertad.
Revista Iberoamericana de Automática e Informática Industrial RIAI, 12(2), 177-188.

Dullerud, G. \& Paganini, F. (2002). A Course in Robust Control Theory: a Convex Approach. Champaign, IL: University of Illinois.

Gahinet, P., Nemirovski, A., Laub, A. \& Chilali, M. (2008). LMI Control Toolbox User's Guide Version 1 for use to MATLAB. Ciudad: Math Works Inc.

Guzmán, L. S., Cardozo, C. A. C. \& Cárdenas, C. A. G. (2014). Diseño e implementación de una plataforma experimental de dos grados de libertad controlada por dos técnicas: pid y lógica difusa. Ciencia e Ingeniería Neogranadina, volumen 24(1), pag 99-115.

Ibáñez, A. (2014). Modelado, identificación, simulación y control óptimo de un helicóptero con dos grados de libertad, cabeceo y guiñada Doctoral dissertation. Universidad Politecnica de Valencia.

Kebede, G. (2006). Robust Decentralized Control of Power Systems: A Matrix Inequalities Approach. These Doktors der Ingenieurwissenschaften.

Universität Duisburg-Essen \& Quanser Inc. (2006). Quanser 2 DOF Helicopter User and Control Manual. Duisburg-Essen, Deutschland: Universität Duisburg-Essen.

Salamanca, J., Díaz, H. \& Higuera, O. (2007). Diseño de un controlador $\mathrm{LMI}+\mathrm{I}$ basado en optimización para un helicóptero de dos grados de libertad. Revista Ingeniería, Investigación y Desarrollo, 5(2), págs. 22-28.

Skogestad, S. \& Postlehwaite, I. (2001). Multivariable Feedback Control Analysis and Design. New York, John Wiley \& Sons (ed). 